r ScIDioc

\section{Accuracy Assessment of a Novel Milling Technique for the Fabrication of Computer Aided Surgical Stents}

Research Article

Heba E. Khorshid ${ }^{1 *}$, Omar Sokker ${ }^{2}$

${ }^{1}$ Assistant Professor, Department of Prosthodontics and Implantology, Faculty of Oral and Dental Medicine, Cairo University, Cairo, Egypt.

2 3D Modeling Specialist, Master's Degree of Applied Arts, Bachelor of Applied Arts, Faculty of Applied Arts, Cairo University, Cairo, Egypt.

\title{
Abstract
}

Purpose: The Target of this work was to compare the amount of both linear and angular deviation of the preplanned implants with the actually placed implants using this new milling technique for the fabrication of computer guided stents which aims to reduce accumulated errors and improve accuracy.

Materials and Methods: This In-vitro study involved the construction of a total of twenty radiographic templates which were transformed into the Computer Aided Surgical Stent (CASS) by simply drilling the implant vector holes into the stent utilizing a CAD/CAM machine rather than using the rapid prototyping technology. Different types of deviations were recorded; the Apical Global, Coronal Global, Apical Vertical, Coronal Vertical, Apical Medio-Lateral, Coronal Medio-Lateral, Apical Antero-posterior and the Coronal Antero-Posterior deviation.

Results: By using one sample t-test and hypothesized population mean $=0$ for both Angular and linear deviations, it was revealed that there were significant differences for all implant deviations as P-value $\leq 0.001$ for all the measurements.

Conclusions: This technique successfully reduced the implant deviation in addition to eliminating expensive machinery for the computer guided stent fabrication thus saving time, material, energy and hence money.

Keywords: Implant Deviation; CAD/CAM; Milling; Computer Guided Surgery; Surgical Guide.

Abbreviations: CASS: Computer Aided Surgical Stent; CAD/CAM: Computer Aided Design/ Computer Aided Manufacturing; CBCT: Cone Beam Computerized Tomography; PP: Pre-planned Implant; AP: Actually Placed Implant; AG: Apical Global Deviation; CG: Coronal Global Deviation; AV: Apical Vertical Deviation; CV: Coronal Vertical Deviation; AML: Apical Medio-Lateral Deviation; CML: Coronal Medio-Lateral Deviation; AAP: Apical Antero-Posterior Deviation; CAP: Coronal Antero-Posterior Deviation.

\section{Introduction}

Historically, surgeons placed implants where the greatest amount of bone was present, without regard to placement of the final restoration. This often led to a compromised final prosthesis with a jeopardized occlusal scheme, poor esthetics and unfavorable biomechanics. The need for more than just osseo-integrated implants led to the evolution of the "Prosthetic-driven implant placement" concept which recommends the placement of implants in the position that serves both functional and esthetic requirements. $1 \mathrm{~A}$ perfect site would be the one that contains sufficient bone volume and serves the ideal prosthetic considerations $[1,2]$. The goal of computer-aided implant planning and placement system is the achievement of the maximal safety, allow implant placement in less time, less postoperative bleeding and discomfort, accelerated recovery for the patient, fewer changes in crestal bone level, reduced tissue trauma and avoiding esthetic failures [3, 4]. However, the use of such technique raised important questions; how accurate is the computer-guided implant placement? Is this new technique safe, efficient and effective to be used in routine clinical settings?. [5]

Lee et al., [6] reported that STL file data are able to accurately fabricate a stereo-lithiographic model and surgical guide for implant surgery. The accuracy of the entire procedure can be measured by

\section{*Corresponding Author:}

Dr. Heba Ezzeldin Khorshid BDS, MS, PhD,

Assistant Professor, Department of Prosthodontics and Implantology, Faculty of Oral and Dental Medicine, Cairo University, Cairo, Egypt.

Tel: $+1310-888-8842$

E-mail: hebakhorshid@hotmail.com/heba.khorshid@dentistry.cu.edu.eg

Received: December 07, 2020

Accepted: January 04, 2021

Published: January 12, 2021

Citation: Heba E. Khorshid, Omar Sokker. Accuracy Assessment of a Novel Milling Technique for the Fabrication of Computer Aided Surgical Stents. Int J Dentistry Oral Sci. 2021;8(1):1356-1361. doi: http://dx.doi.org/10.19070/2377-8075-21000268

Copyright: Heba Ezzeldin Khorshid ${ }^{\circ} 2021$. This is an open-access article distributed under the terms of the Creative Commons Attribution License, which permits unrestricted use, distribution and reproduction in any medium, provided the original author and source are credited. 
the "deviation between the position of the actual implant and the planned one". This deviation usually happen as result of the sum of errors that accumulate throughout the computer-aided cascade, starting from scanning, processing, planning, till the surgical and prosthetic phase [7]. The intense need for finding a solution to those inevitable deviations in the literature due to the added and accumulated errors of the numerous steps of the computer guided surgical stent fabrication was the main reason behind the innovation of this idea.

\section{Materials and Methods}

The construction of this novel Computer aided surgical stent (CASS) involved the use of three main tools: 1 . The Orienter, 2. The Orienter Mount and 3. The Fixation Plane Metal Disc.

For this In-vitro study, a total of twenty radiographic templates on twenty different maxillary casts were constructed; the sample size calculation was carried out by a "power and sample size" program (G*Power program, University of Düsseldorf). The initial step involved the construction of satisfactory conventional complete dentures for the twenty maxillary casts followed by duplication of the maxillary dentures. For the denture base, clear heat cured acrylic resin (Meliodent.Heraeus-Kulser $\mathrm{GmbH}$ ) was used while a mixture of sifted barium sulphate and clear heat cured acrylic resin in a ratio of 1:3 respectively was used for the teeth only as seen in Fig.1A. After adequate finishing and polishing of the radiographic template, $2 \mathrm{~mm}$ channels were drilled through the stent at the central axes of the lateral incisor, first premolar and first molar region which were then filled with self-cured clear acrylic resin.

The following work flow was performed to construct the Computer Aided Surgical Stent (CASS):

Orienter preparation and attachment to the radiographic Template

The main purpose of the Orienter as seen in Fig.1B and $1 \mathrm{C}$ was that it acted as the main reference to which the milling machine will be able to identify the spatial position of the pre-planned im- plant vectors and hence the $(x-y-z)$ point of entry of each planned implant site. It also fixed and stabilized the CASS to the milling machine disc during the milling procedure. The Orienter contains twelve rectangular shaped engravings which were filled with light cured composite resin. With the use of medical grade adhesive, the orienter was first secured to the facial aspect of the radiographic template as shown in Fig.1C. A thin mix of self-cured clear acrylic resin was poured to firmly secure the radiographic stent and the orienter together with the aid of the cone shaped retentive aids on the orienter's inner/lingual aspect. The orienter should always be placed with the "O" side facing the occlusal aspect of the Radiographic template and the " $\mathrm{M}$ " facing the mucosal surface of the Radiographic template as seen in Fig.1D.

\section{Orienter positioning on the Orienter Mount}

The projecting Extra-oral male portion of the Orienter was secured over the orienter mount which consisted of a female counterpart. The Orienter mount was attached to the bite block slot of the CBCT machine as seen in Fig.1E and 1F. The orienter mount was calibrated using a sensitive digital laser water balance at a zero-zero-zero $x-y-z$ position. The red midline laser beam of the CBCT machine should exactly coincide with a midline groove engraved on the projecting male portion of the orienter. The purpose of this orienter mount was to automatically place the orienter/patient at a standardized zero-zero-zero $x-y-z$ position thus negating the need to do any registration or superimposition procedure as we already know the orientation of the orienter.

\section{Image acquisition using CBCT}

The model stone casts with the radiographic stents were radiographed using Cone Beam Computed Tomographic (CBCT) scanning machine (Scanora 3D Soredex). The resultant images were obtained as Dicom data on a compact disc.

\section{Virtual Orienter Registration with the Actual/real Orienter}

STL files of the Virtual Orienter/Fixation plane complexes were imported into each of the twenty radiographic template projects loaded on the Mimics software (Mimics, Materialise HQ) as seen

Figure 1. A: Duplicated maxillary dentures / Radiographic template B: The Orienters C: The Orienter being attached to the radiographic template. Note Light cured composite being filled in the rectangular shaped engravings D: Self-cured clear acrylic resin used to firmly secure the radiographic stent and the orienter together with the aid of the cone shaped retentive aids on the orienter's inner/lingual aspect E: Side view of the radiographic stent/orienter complexes mounted over the orienter mount for the in-vitro study for image acquisition F: Occlusal view of the radiographic stent/orienter complexes mounted over the orienter mount for the in-vitro study for image acquisition.

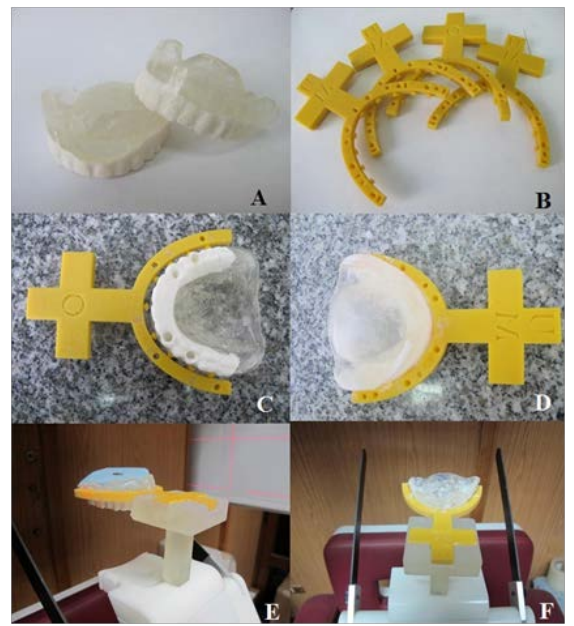


in Fig.2A. The Orienter and Fixation plane were initially designed and created by Rhinoceros (Rhinoceros ${ }^{\circledR}$ ). The Orienter and Fixation plane were already designed and created in a zero-zero-zero $x-y-z$ position such that when imported into any Mimics project, all that was done was dragging it manually over the radio-opaque rectangular composite markers or automatically superimposing it using the STL registration tool available in the Mimics software.

\section{Virtual Implant planning and Exporting STL file extension}

The implant planning was performed for the twenty projects using the Mimics software as seen in Fig.2B. Virtual implant planning was performed at the desired implant sites which were identified through the radiolucent channels previously prepared in the radiographic templates as shown in Fig.2C. Using the Boolean operation tool, the supra bony (sleeve) portions of the virtual implants were united together as shown in Fig.2D which were then all united with the virtual fixation plane as revealed in Fig.2E. The rationale behind this was to obtain the implant vectors represented by the supra-bony (sleeve) portions in relation to the fixation plane. The Fixation plane was designed such that it had a constant spatial relationship with the CAD/CAM machine disc. The resultant union object was then exported as an STL file extension from the mimics program as shown in Fig. $2 \mathrm{~F}$.
Fixation of the Orienter on The Fixation Plane within CAD/ CAM Milling machine

The Radiographic template at this point of work is now denoted the CASS. The Extra-oral portion of the Orienter was disked away as seen in Fig.3A then the Orienter/CASS complex was securely anchored with the fixation plane using four bolts and nuts that pass through coinciding four holes created in both the orienter and fixation plane metal disc as illustrated in Fig.3B.

CAM Software and Milling Procedure within the radiographic template

The resultant STL object (union of the Supra-bony sleeves with the fixation plane) exported from the mimics program was then imported into the CAM software of the CAD/CAM milling machine. The Fixation plane was designed such that it has a constant spatial relationship with the CAD/CAM machine disc. Accordingly, the vectors (supra-bony sleeves) of each implant can now be identified by the CAM software. The CAM software will begin generating a cascade of tasks that will be sent to the milling machine. Manual cancellation of all the operations to be done was performed while leaving only the vector holes of the supra-bony sleeves as shown in Fig. 3C. Subsequently, the resultant task sent to the Milling machine was drilling of the vector holes into the

Figure 2. A: Radiographic markers in the Orienters being superimposed. B: Virtual implant planning being performed at the desired implant sites. C: Virtual planning of the six implants in relation to the fixation plane. D: Using the Boolean operation tool, the supra bony (sleeve) portions of the virtual implants were first separated from implants bodies then united together. E: United sleeves being united with the fixation plane/orienter complex. F: Stl object being exported from the Mimics software.

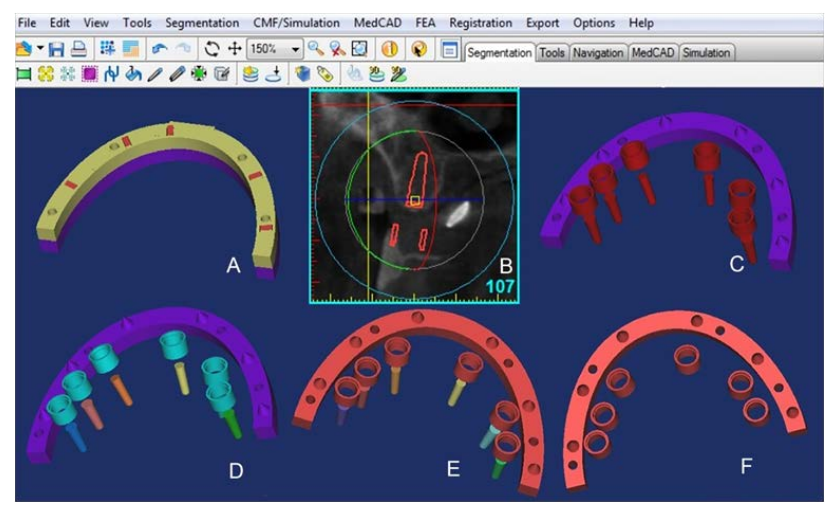

Figure 3. A: The Extra-oral portion of the orienter being disked away B: The Orienter/ ICASS complex being firmly secured with the fixation plane disc which is anchored to the CAD/CAM Machine disc receptacle C: STL file being imported into the Cam software which will identify the vector sleeve holes of the planned implants D: Milling of the vector holes of the preplanned implants directly on the ICASS.

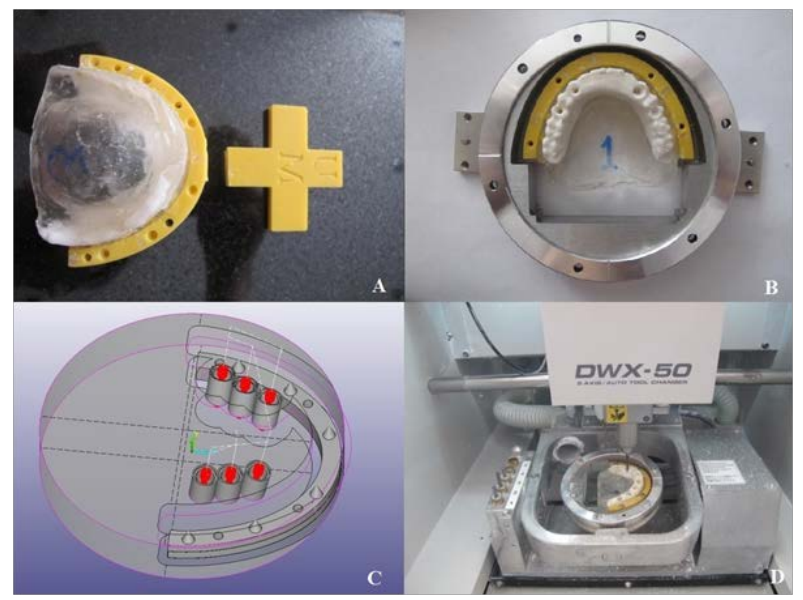


ICASS. The Roland DWX50 CAD/CAM machine (Roland DG Corporation, Hamamatsu-shi, Shizuoka-ken Japan) took from 3-5 minutes for drilling each vector hole as seen in Fig. 3D. Metal Sleeves were then bonded to the drill holes.

\section{Implant Deviation Analysis}

To analyze the Deviation of the Preplanned PP Implant positions from the Actually Placed AP Implants, Model implants were secured and accurately fit into the metal sleeves to simulate the actually placed implants' positions. The CASS were then radiographed using the CBCT scanning machine to obtain a new post-operative simulated project. Using the composite resin radio-opaque markers, Superimposition of the PP project orienters with the new post-operative project was performed using the Mimics software STL registration tools as seen in Fig. 4A. The STL objects of the $\mathrm{PP}$ and AP were then exported to the software Rhinoceros for further analysis and interpretation as shown in Fig. 4B.

\section{Results}

This study was performed to assess the amount of both linear and angular deviation of the actually placed AP implants in comparison with the virtually PP implants using this new technique for the fabrication of computer guided stents. Different types of deviations were recorded using the computer software Rhinoceros; the Apical Global AG, Coronal Global CG, Apical Vertical AV, Coronal Vertical CV, Apical Medio-Lateral AML, Coronal Medio-Lateral CML, Apical Antero-posterior AAP and the Coronal Antero-Posterior CAP deviation.

Values recorded were presented as mean and standard deviation (SD) values and further statistical analysis was performed for the In-vitro study as seen in Fig. 5 and Gand Table 1.

Using Kolmogorov-Smirnov and Shapiro-Wilk tests, data meas- urements revealed parametric distribution. Standard one sample t-test followed by one way ANOVA test for overall comparison between all measurements was performed. Mood's Median Test was also used to assess the number of observations in each sample on either side of the grand median calculated for both the Angular and the linear deviation. By using one sample t-test and hypothesized population mean $=0$ for both Angular and linear deviations, it was revealed that there were significant differences for all implant deviations as $\mathrm{P}$-value $\leq 0.001$ for all the measurements. The results for ANOVA comparison between the Linear deviations was statistically significant between the groups (F-value $=30.294$, P-value $\leq 0.001)$. Meanwhile the comparison between the $\mathrm{X}$-axis and $\mathrm{Y}$-axis deviations of the implant showed a statistically insignificant difference $(\mathrm{t}$-value $=0.845, \mathrm{P}$-value $=0.399)$ as seen in Table 1.

\section{Discussion}

In the current study, results revealed that the amount of mean angular deviation between the PP and the AP implants were $0.965^{\circ}$ and $1.064^{\circ}$ in the $\mathrm{x}$-axis and $\mathrm{y}$-axis respectively. Results also revealed that the amount of mean linear deviation in the AG, CG, AV, CV, AML, CML, AAP and the CAP planes were 0.471, 0.484, $0.275,0.285,0.278,0.247,0.259$ and $0.241 \mathrm{~mm}$ respectively as seen in Fig. 5.

In a Meta-analysis conducted by Van Assche et al., [8] study revealed a mean error of $0.99 \mathrm{~mm}$ (ranging from 0 to $6.5 \mathrm{~mm}$ ) at the entry point and of $1.24 \mathrm{~mm}$ (ranging from 0 to $6.9 \mathrm{~mm}$ ) at the apex. The mean angular deviation was $3.81^{\circ}$ (ranging from 0 to $24.9^{\circ}$ ). Another meta-analysis performed by Tahmseb at al. [9] to study the accuracy of implant placement ( 24 clinical and preclinical studies) revealed a total mean error of $1.12 \mathrm{~mm}$ (maximum of $4.5 \mathrm{~mm}$ ) at the entry point measured in 1,530 implants and 1.39 $\mathrm{mm}$ at the apex (maximum of $7.1 \mathrm{~mm}$ ) measured in 1,465 im-

Figure 4. A: Superimposition of the STL files of the PP with the Actually Placed (AP) implants using the Orienter/fixation plane for the in-vitro study B: Linear and Angular deviation being analysed using the Rhinoceros program for the in-vitro study.

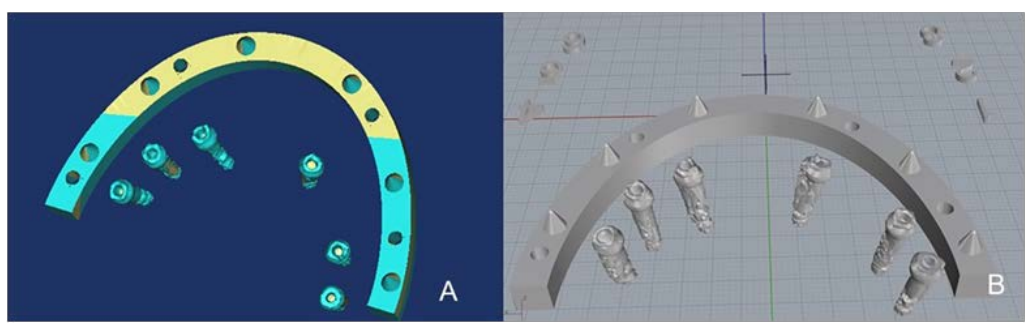

Figure 5. A bar chart showing mean implant angular (X-axis and $\mathrm{Y}$-axis) and Linear deviations (Apical Global AG, Coronal Global CG, Apical Vertical AV, Coronal Vertical CV, Apical Medio-Lateral AML, Coronal Medio-Lateral CML, Apical Antero-posterior AAP and Coronal Antero-Posterior CAP deviation).

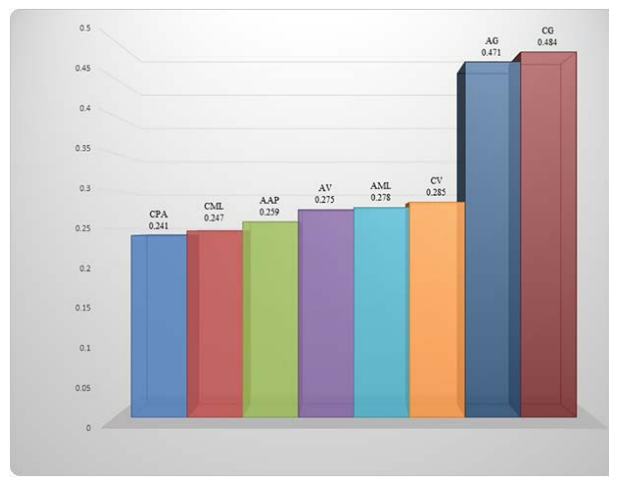


Figure 6. A box plot for the implant angular (X-axis and $\mathrm{Y}$-axis) and Linear deviations.

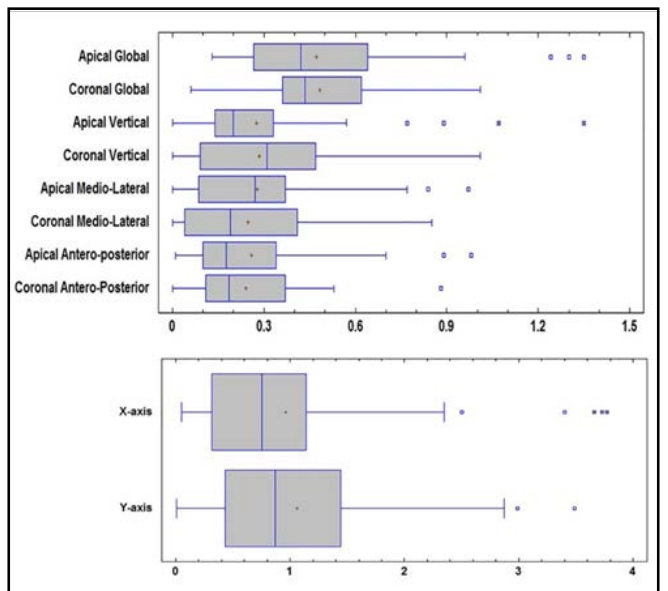

Table 1. Mean, standard deviation (SD), standard error of mean (SEM) and Median of measurements taken for both Angular and Linear implant deviations for the In-vitro study.

\begin{tabular}{|c|c|c|c|c|c|c|c|c|c|}
\hline & & \multicolumn{8}{|c|}{ In-Vitro Study } \\
\hline \multicolumn{2}{|r|}{ Deviation } & Mean & SD $†$ & SEMS & Min & Max & Median & $\begin{array}{c}\text { T- } \\
\text { Value }\end{array}$ & P-value \\
\hline \multirow{2}{*}{$\begin{array}{l}\text { Angular } \\
\text { (degree) }\end{array}$} & $\mathrm{X}$-axis & 0.965 & 0.941 & 0.086 & 0.05 & 3.77 & 0.75 & 11.235 & \multirow{10}{*}{$\leq 0.001 *$} \\
\hline & $\mathrm{Y}$-axis & 1.064 & 0.867 & 0.079 & 0.01 & 3.49 & 0.87 & 13.431 & \\
\hline \multirow{8}{*}{$\begin{array}{l}\text { Linear } \\
(\mathrm{mm})\end{array}$} & Apical Global & 0.471 & 0.267 & 0.025 & 0.13 & 1.35 & 0.42 & 19.302 & \\
\hline & Coronal Global & 0.484 & 0.207 & 0.019 & 0.06 & 1.01 & 0.435 & 25.595 & \\
\hline & Apical Vertical & 0.275 & 0.234 & 0.021 & 0 & 1.35 & 0.2 & 12.869 & \\
\hline & Coronal Vertical & 0.285 & 0.23 & 0.021 & 0 & 1.01 & 0.31 & 13.544 & \\
\hline & Apical Medio-Lateral & 0.278 & 0.214 & 0.020 & 0 & 0.97 & 0.27 & 14.217 & \\
\hline & Coronal Medio-Lateral & 0.247 & 0.239 & 0.022 & 0 & 0.85 & 0.19 & 11.325 & \\
\hline & Apical Antero-posterior & 0.259 & 0.226 & 0.021 & 0.01 & 0.98 & 0.175 & 12.55 & \\
\hline & Coronal Antero-Posterior & 0.241 & 0.161 & 0.015 & 0 & 0.88 & 0.185 & 16.384 & \\
\hline
\end{tabular}

a Significant at $\mathrm{P} \leq 0.05$

* Standard Deviation

† Standard Error of Mean

plants. This was in accordance with another systematic review of literature performed by D'Haese et al., [10] to assess the accuracy and complications using Computer-Designed Surgical Guides which revealed that guided implant surgery was far from accurate when using computer designed stereolithographic surgical guides.

In a clinical study performed by Pattersson et al., [11] results revealed that there were significant differences between virtually planned implants' position and the final position of clinically placed implant. Another study published by Turbush and Turkyilmaz, [12] showed that the mean linear deviation of the long axis of implants placed using Sterolithiographic SLA guides in thirty mandibles between the planned and placed implants were $1.18 \pm$ $0.42 \mathrm{~mm}$ at the implant neck and $1.44 \pm 0.67 \mathrm{~mm}$ at the implant apex for all 150 implants. Results also showed that the mean angular deviation of the long axis between the planned and placed implants was $2.2^{\circ} \pm 1.2^{\circ}$. On the other hand, in a study conducted by Beretta et al.,13 the comparison of 14 implants showed a lower mean linear deviation values than the previous studies; which was $0.56 \pm 0.23 \mathrm{~mm}$ at the implant head and $0.64 \pm 0.29 \mathrm{~mm}$ at the implant apex, and a mean angular deviation of the long axis of $2.42^{\circ} \pm 1.02^{\circ}$. As shown in the previous studies, the amount of implant deviations from the PP to the AP using SLA computer guided stents were in some instances considerably high and could have led to detrimental effects on the surrounding vital structures. Furthermore, major prosthetic errors could occur if immediate functional loading using prosthesis generated from computer guided preoperative plans were to be performed.

\section{Conclusion}

The Roland DWX50 CAD/CAM machine took from 3-5 minutes to drill each vector hole which meant that we saved tremendous time as well as energy with no need for any extra material and money hence a greener earth. This novel technique also helped eliminate unnecessary errors by simply cutting on the numerous steps involved in constructing any conventional computer guided surgical stents. Another great advantage was utilizing the accuracy of fit, adaptation, retention and stability already achieved in the radiographic template hence reducing the errors that might occur from misfit of SLA templates. 


\section{Disclosure}

The authors claim to have no financial interest in any company or any of the products mentioned in this article. This research did not receive any specific grant from funding agencies in the public, commercial, or not-for-profit sectors.

\section{References}

[1]. Kopp KC, Koslow AH, Abdo OS. Predictable implant placement with a diagnostic/surgical template and advanced radiographic imaging. J Prosthet Dent 2003;89:611-5.PubmedPMID: 12815357.

[2]. Widmann G, Bale RJ. Accuracy in computer-aided implant surgery--a review. Int J Oral Maxillofac Implants. 2006;21:305-13.PubmedPMID: 16634503.

[3]. Widmann G ,Zangerl A, Keiler M, Stoffner R, Bale R, Puelacher W. Flapless implant surgery in the edentulous jaw based on three fixed intraoral reference points and image-guided surgical templates: accuracy in human cadavers. Clin Oral Implants Res 2010;21,835-41. PubmedPMID:20345381.

[4]. Vieira DM, Sotto-Maior BS, Barros CAV de S, Reis, ES, Francischone CE. Clinical accuracy of flapless computer-guided surgery for implant placement in edentulous arches. Int J Oral Maxillofac Implants 2013;28,1347-51.PubmedPMID:24066327.

[5]. Valente F, Schiroli G, Sbrenna A. Accuracy of computer-aided oral implant surgery: a clinical and radiographic study. Int J Oral Maxillofac Implants 2009;24,234-42.PubmedPMID: 19492638.
[6]. Lee CY, Ganz SD, Wong N, Suzuki JB.Use of cone beam computed tomography and a laser intraoral scanner in virtual dental implant surgery: part 1. Implant Dent 2012; 21(4):265-71.PubmedPMID:22814549.

[7]. Koop R, Vercruyssen M, Vermeulen K, Quirynen M. Tolerance within the sleeve inserts of different surgical guides for guided implant surgery. Clin Oral Implants Res 2013;24,630-634.PubmedPMID:22413853.

[8]. Van Assche N, Vercruyssen M, Coucke W, Teughels W, Jacobs R, Quirynen M. Accuracy of computer-aided implant placement. Clin. Oral Implants Res. 2012,; 23(6):112-123.PubmedPMID:23062136.

[9]. Tahmaseb A, Wismeijer D, Coucke W, M, Derksen W. Computer technology applications in surgical implant dentistry: a systematic review. Int J Oral Maxillofac Implants 2014;29:25-42. PubmedPMID:19885437.

[10]. D'Haese J, Van De Velde T, Komiyama A, Hultin M, De Bruyn H. Accuracy and complications using computer-designed stereolithographic surgical guides for oral rehabilitation by means of dental implants: a review of the literature. Clin Implant Dent Relat Res 2012;14:321-35. PubmedPMID:20491822.

[11]. Pettersson A, Komiyama A, Hultin M, Näsström K, Klinge B. Accuracy of Virtually Planned and Template Guided Implant Surgery on Edentate Patients. Clin Implant Dent Relat Res 2012;14:527-37. PubmedPMID:20491812.

[12]. Turbush SK, Turkyilmaz I. Accuracy of three different types of stereolithographic surgical guide in implant placement: an in vitro study. J Prosthet Dent 2012;108:181-8. PubmedPMID:22944314.

[13]. Beretta M, Poli PP, Maiorana C. Accuracy of computer-aided templateguided oral implant placement: a prospective clinical study. J Periodontal Implant 2014;44:184-93.PubmedPMID: 25177520 